\title{
A putative high affinity phosphate transporter, CmPT1, enhances tolerance to Pi deficiency of chrysanthemum
}

Peng Liu ${ }^{1,2}$, Sumei Chen ${ }^{1}$, Aiping Song ${ }^{1}$, Shuang Zhao ${ }^{1}$, Weimin Fang ${ }^{1}$, Zhiyong Guan ${ }^{1}$, Yuan Liao ${ }^{1}$, Jiafu Jiang ${ }^{1 *}$ and Fadi Chen ${ }^{1,2^{*}}$

\begin{abstract}
Background: Inorganic phosphate (Pi) is essential for plant growth, and phosphorus deficiency is a main limiting factor in plant development. Its acquisition is largely mediated by Pht1 transporters, a family of plasma membrane-located proteins. Chrysanthemum is one of the most important ornamental plants, its productivity is usually compromised when grown in phosphate deficient soils, but the study of phosphate transporters in chrysanthemum is limited.

Results: We described the isolation from chrysanthemum of a homolog of the Phosphate Transporter 1 (PT1) family. Its predicted product is a protein with 12 transmembrane domains, highly homologous with other high affinity plant Pi transporters. Real-time quantitative PCR analysis revealed that the gene was transcribed strongly in the root, weakly in the stem and below the level of detection in the leaf of chrysanthemum plants growing in either sufficient or deficient Pi conditions. Transcript abundance was greatly enhanced in Pi-starved roots. A complementation assay in yeast showed that $\mathrm{CmPT1}$ partially compensated for the absence of phosphate transporter activity in yeast strain MB192. The estimated $K_{m}$ of $\mathrm{CmPT1}$ was $35.2 \mu \mathrm{M}$. Under both Pi sufficient and deficient conditions, transgenic plants constitutively expressing CMPT1 grew taller than the non-transformed wild type, produced a greater volume of roots, accumulated more biomass and took up more phosphate.
\end{abstract}

Conclusions: CMPT1 encodes a typical, root-expressed, high affinity phosphate transporter, plays an important role in coping Pi deficiency of chrysanthemum plants.

Keywords: Chrysanthemum morifolium, CmPT1, Functional characterization, Complementation assay, Transgenic plants

\section{Background}

After nitrogen, phosphorus is the most frequently limiting plant macronutrient [1]. Despite its abundance in the soil, most of it is present in a form which is not readily accessible to plants [2]. The application of phosphate-based fertilizer can correct deficiency, but even when provided in a highly accessible form, as little as $10 \%$ of the phosphorus applied is recovered by plants [3], with the remainder either becoming immobilized or lost through run-off [4]. The availability of economically viable sources

\footnotetext{
* Correspondence: jiangjiafu@njau.edu.cn; chenfd@njau.edu.cn ${ }^{1}$ College of Horticulture, Nanjing Agricultural University, Nanjing 210095, China

${ }^{2}$ Jiangsu Province Engineering Lab for Modern Facility Agriculture Technology \& Equipment, Nanjing 210095, China
}

of phosphate fertilizer is declining [5]. There is thus some urgency associated with efforts to promote the efficiency with which crop plants are able to take up phosphate.

The concentration of inorganic phosphate $(\mathrm{Pi})$ in plant tissues lies in the range $5-20 \mathrm{mM}$ [6], whereas the level available in a typical soil is three orders of magnitude lower than this [7]. Thus the acquisition of Pi occurs against a steep concentration gradient [8]. Pi uptake by plants operates via both a low and a high affinity system $[7,9]$. The high affinity route is largely mediated by plasma membrane-localized $\mathrm{Pi}$ transporters belonging to the PHOSPHATE TRANSPORTER1 (Pht1) family $[8,10]$. Following the identification of Pi transporters in Arabidopsis thaliana [10], a large number of $P T$ genes have been identified and characterized in a wide range of species such as 
Arabidopsis thaliana [3,11,12], rice [13-15], tomato [16], tobacco [17,18], maize [19], barley [20], Medicago truncatula [21], Populus trichocarpa [22], and soybean [23].

The chrysanthemum (Chrysanthemum morifolium Ramat.) is a leading ornamental species. Its productivity is usually compromised when grown in phosphate deficient soils. Here, we describe the isolation of a Pht1 homolog present in the chrysanthemum variety 'Nannongyinshan', a cultivar which is relatively tolerant of phosphate deficiency [24]. The gene's transcription profile was characterized and the beneficial effect of its constitutive expression on the plant's ability to cope with phosphate deficiency was demonstrated.

\section{Methods}

\section{Plant material and growing conditions}

Cuttings of the chrysanthemum cultivar 'Nannongyinshan' were obtained from the Chrysanthemum Germplasm Resource Preserving Centre, Nanjing Agricultural University, China, 'Nannongyinshan' was a low phosphorus tolerant cultivar in our privious study, and 'Jinba' is a low phosphorus intolerant cultivar compared to 'Nannongyinshan' [24]. The cuttings were raised in a greenhouse in a 1:1 mixture of perlite and vermiculite, without any fertilizer supplementation. After two weeks, the plants were up-rooted, their roots were washed free of the rooting medium and the plants were transferred to a hydroponic solution consisting of a diluted (1:2) Hoagland's solution [25]. The phosphate treatments were initiated one week after the transfer, by removing three plants to an aerated hydroponic solution containing either $300(+\mathrm{P})$ or $0(-\mathrm{P})$ $\mu \mathrm{M} \mathrm{Pi}$. The nutrient solution was replaced every three days. Leaf, stem and root tissue was harvested after a further 11 days, snap-frozen in liquid nitrogen and stored at $-80^{\circ} \mathrm{C}$.

\section{Isolation of CmPT1}

The design of a pair of degenerate PCR primers (DPF/R; sequences given in Table 1) was based on a peptide alignment of the Pht1 sequences of Arabidopsis thaliana (GenBank accession number AED94948), Capsicum frutescens (ABK63958), Lupinus albus (AAK01938), Hordeum vulgare (AAN37900) and Petunia $x$ hybrida (ABS12068). Total RNA was extracted from the roots of P0 treated 'Nannongyinshan' plants using the RNAiso reagent (TaKaRa, Japan). A full length chrysanthemum cDNA sequence (deposited in GenBank as accession KC812501) was isolated using RACE technology, following [26]. The RACE primer sequences are given in Table 1.

Table 1 Adaptor and primer sequences used

\begin{tabular}{|c|c|c|}
\hline Primer name & $5^{\prime}-3$ ' sequence & Usage \\
\hline Oligo d $(T)_{18}$ & TTाTाTाTाTाTाTाTा & Reverse transcription \\
\hline DPF & TGGCGGATGAAGATGCCNGARAC & Degenerated PCR \\
\hline DPR & ACCGGGCGGGGAADATYTCNGC & Degenerated PCR \\
\hline AAP & GGCCACGCGTCGACTAGTACGGG\|GGG\|GGG\|G & $5^{\prime}-\mathrm{RACE}$ \\
\hline AUAP & GGCCACGCGTCGACTAGTAC & $5^{\prime}-\mathrm{RACE}$ \\
\hline GSP5'-1 & TCCGAGCAAGTGAAGG & $5^{\prime}-\mathrm{RACE}$ \\
\hline GSP5'-2 & AACCCAAATGAGTTGCTCTTGTCC & $5^{\prime}-$ RACE \\
\hline GSP5'-3 & GCCATATCTTGTGCAGCTTGT & $5^{\prime}-\mathrm{RACE}$ \\
\hline dT-B26 & GACTCGAGTCAACATCGATTITITIITIIITITIT & $3^{\prime}-$ RACE \\
\hline B26 & GACTCGAGTCAACATCGA & $3^{\prime}-$ RACE \\
\hline GSP3'-1 & GTGCTACCGGCGAGGTTTAT & $3^{\prime}-\mathrm{RACE}$ \\
\hline GSP3'-2 & GTCCCTGGTTACTGGTTCACTG & $3^{\prime}-$ RACE \\
\hline Full-F & ATCTCCTTTTCTTCAATCACTCTCT & ORF amplifications \\
\hline Full-R & TCTAGGGTTITATTCTTCATACCCT & ORF amplifications \\
\hline CMPT1-EcoR-F & CCGGAATTCCGGGTTTCGAAGGAGGAGTGTATCCATG & Functional complementation \\
\hline CmPT1-Not-R & ATTTGCGGCCGCAACAGTATTCAGTCAATCAAGCCGG & Functional complementation \\
\hline qGSP-F & CTCGCTCGTTGTTCTTGGTATC & qRT-PCR \\
\hline qGSP-R & AACAGTATTCAGTCAATCAAGC & qRT-PCR \\
\hline EF1A-F & TITTGGTATCTGGTCCTGGAG & qRT-PCR \\
\hline EF1A-R & CCATTCAAGCGACAGACTCA & qRT-PCR \\
\hline $35 S-F$ & AGATACAGTCTCAGAAGACCAAAGG & Transgenic detection \\
\hline $35 S-R$ & TTGATATTCTTGGAGTAGACGAGAG & Transgenic detection \\
\hline
\end{tabular}




\section{Sequence analysis}

The open reading frame (ORF) of the full length cDNA isolated from 'Nannongyinshan' was identified using the ORF finder program (DNASTAR. Lasergene. v7.1). The location of hydrophobic and putative transmembrane domains was enabled through the software package mounted at http://expasy.org/tools/protscale.html. Multiple peptide alignments were carried out using DNAman software (v5.2.2.0; Lynnon Biosoft, St Louis, QC, Canada), and phylogenetic analyses using Clustal X and MEGA v4.0 software.

\section{CIPT1 transcription profiling}

Total RNA was extracted from root, stem and leaf tissue of plants grown both in the $+\mathrm{P}$ and $-\mathrm{P}$ treatments using the RNAiso reagent, and was then used as the template for real-time quantitative PCR (qRT-PCR) assays, based on the SYBR Green master mix (SYBR Premix Ex Taq ${ }^{\text {Ts }}$ II, TaKaRa Bio) and the gene-specific primer pair qGSPF/-R (sequences given in Table 1), which generated a $163 \mathrm{bp}$ fragment. The reference sequence was a $151 \mathrm{bp}$ fragment of $E F-1 a$, amplified with the primers EF1a $-F /-\mathrm{R}$ (sequences given in Table 1). Each $20 \mu \mathrm{l}$ RT-qPCR contained $10 \mu \mathrm{l}$ SYBR Green master mix, $10 \mathrm{ng}$ cDNA template and $0.2 \mu \mathrm{M}$ of each primer. The PCR program comprised an initial denaturation step $\left(95^{\circ} \mathrm{C} / 60 \mathrm{~s}\right)$, followed by 40 cycles of $95^{\circ} \mathrm{C} / 15 \mathrm{~s}, 60^{\circ} \mathrm{C} / 15 \mathrm{~s}, 72^{\circ} \mathrm{C} / 45 \mathrm{~s}$. Relative transcription levels were estimated using the $2^{-\Delta \Delta C t}$ method [27].

\section{Complementation of a yeast mutant strain defective for Pi uptake}

The function of the chrysamthemum gene (denoted CmPT1) was studied by its heterologous expression in yeast strain MB192 (MATa pho3-1 Dpho84::HIS3 ade2 leu2-3.112 his3-532 trp1-289 ura3-1.2 can1) which lacks phosphate transporter activity. First, the CmPT1 ORF was amplified using a Phusion High-Fidelity PCR kit (New England Biolabs, lpswich, MA, USA) based on the primer pair CmPT1-EcoR-F/Not-R (sequences given in Table 1), and the resulting amplicon was digested with EcoRI and NotI and then introduced into the yeast

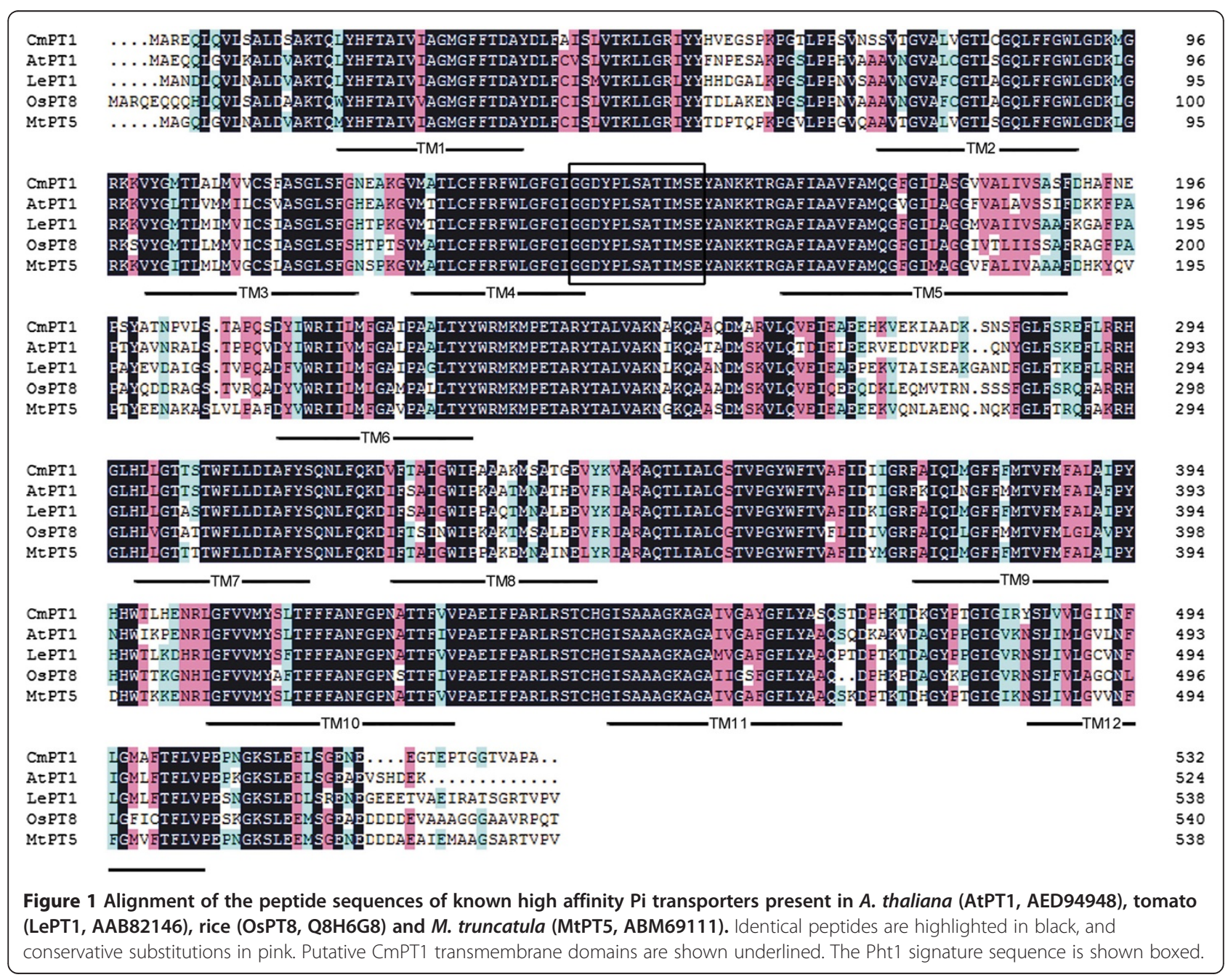


expression vector p112A1NE. The structure of the resulting recombinant plasmid (p112A1NE-CmPT1) was confirmed by restriction enzyme digestion and DNA sequencing. MB192 cells were transformed with either p112A1NE-CmPT1 or with the empty p112A1NE vector. Transgenic cells were grown in a yeast nitrogen base (YNB) medium until up to the logarithmic phase, and the medium was then replaced with a range of $\mathrm{Pi}$ concentrations $(20,60$ and $100 \mu \mathrm{M})$ and the cells left to grow for a further $20 \mathrm{~h}$. Bromocresol Purple was used to indicate the $\mathrm{pH}$ of the medium, giving a color shift, from yellow to purple, during the acidification of the liquid medium: this change correlated well with the growth of the yeast cells [14]. Thereafter, the cells were transferred into YNB medium containing $60 \mu \mathrm{M}$ Pi for a further $40 \mathrm{~h}$. The optical density of the yeast cultures was measured every $8 \mathrm{~h}$. The $\mathrm{pH}$ dependence of Pi uptake was studied by growing the cells in a series of MES-based YNB buffers containing $60 \mu \mathrm{m} \mathrm{Pi}$ at a $\mathrm{pH}$ of between 4 and 8 . The kinetic properties of CmPT1 were analysed by feeding the transformed yeast cells with ${ }^{32} \mathrm{P}$ labelled $\mathrm{Pi}$, following the methods described by [13]. The $K_{m}$ value was calculated using GraphPad v4.0 software.

\section{Construction of an constitutive expression vector and the development of transgenic plants}

CmPT1 was inserted into the pBIG vector (offered by Professor Junping Gao, Department of Ornamental Horticulture, China Agricultural University) under the control of the CaMV 35S promoter. The structure of the resulting pBIG-CmPT1 recombinant plasmid was confirmed by restriction enzyme digestion and DNA sequencing, and was then transformed into Agrobacterium tumefaciens strain EHA105 via the heat shock method [28]. The recipient of the transgene was the chrysanthemum cultivar 'Jinba', which is intolerant of phosphate deficiency [24]. Transformation was effected using a slightly modified form of the procedure described by [29]. Explants developing multiple shoots were transferred to a selective regeneration medium, with the addition of $1.0 \mathrm{mg} \cdot \mathrm{L}^{-1}$ 6-BA, $0.5 \mathrm{mg} \cdot \mathrm{L}^{-1} \mathrm{NAA}, 10 \mathrm{mg} \cdot \mathrm{L}^{-1}$ kanamycin and $300 \mathrm{mg} \cdot \mathrm{L}^{-1}$ carbenicillin. Regenerating shoots were then rooted by transferring the material to a half-strength Murashige and Skoog medium containing $7.5 \mathrm{mg} \cdot \mathrm{L}^{-1}$ kanamycin and $200 \mathrm{mg} \cdot \mathrm{L}^{-1}$ carbenicillin. After acclimation, kanamycin-resistant plants were transplanted to soil and grown on in a greenhouse.

\section{The phenotype of transgenic lines constitutively expressing CMPT1}

Verification of transformation was obtained by a PCR assay of genomic DNA isolated from both presumptively transformed rooted plants, primed with the oligonucleotide pair 35 s-F/-R (sequences given in Table 1) which

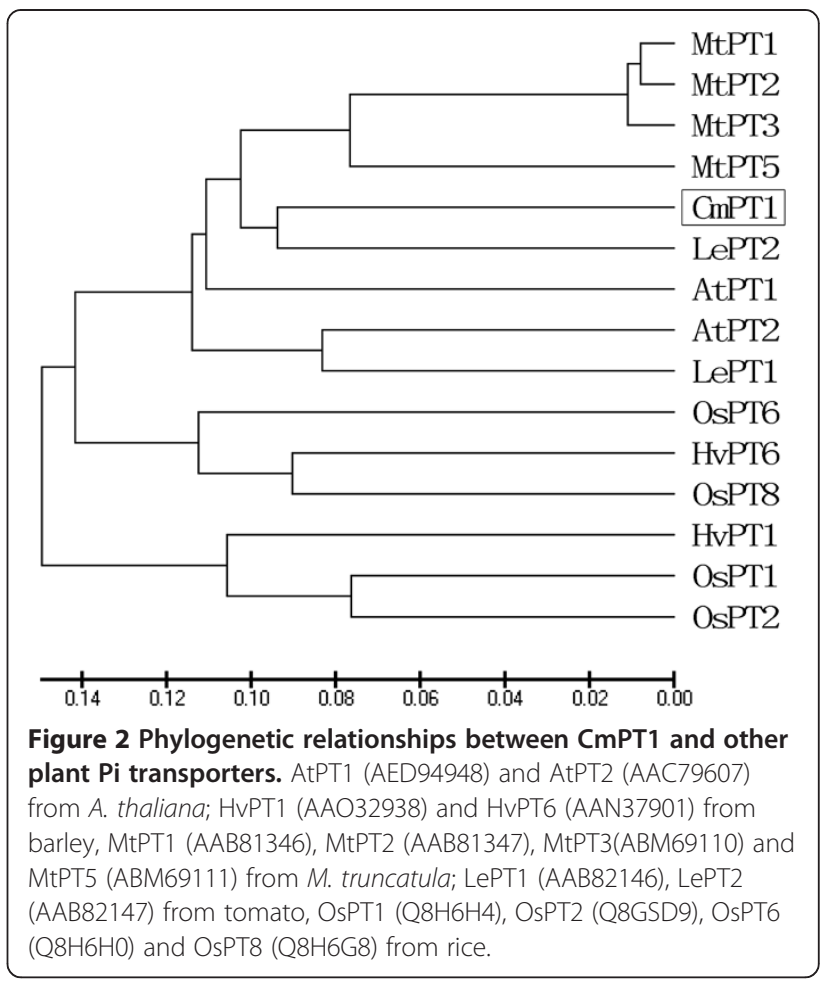

targets the CaMV 35S promoter sequence. The PCR was initiated by a denaturation step $\left(94^{\circ} \mathrm{C} / 5 \mathrm{~min}\right.$, which was followed by 30 cycles of $94^{\circ} \mathrm{C} / 45 \mathrm{~s}, 55^{\circ} \mathrm{C} / 45 \mathrm{~s}, 72^{\circ} \mathrm{C} / 30 \mathrm{~s}$, and completed with a final elongation step of $72^{\circ} \mathrm{C} /$ 10 min. Transcript abundance of CmPT1 in the confirmed

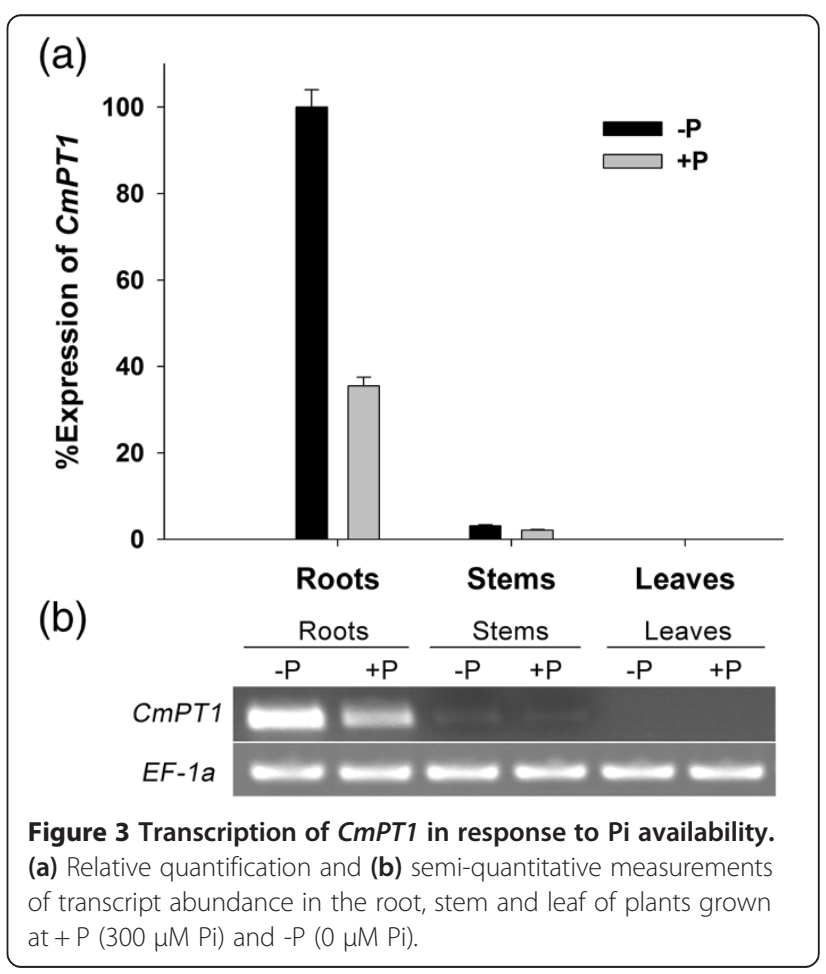


Figure 4 Complementation assay of CmPT1 in yeast strain MB192. (a) Test for acid phosphatase activity. Yp1 12-CmPT1 carries the CMPT1 transgene, Yp1 12 is an empty vector, WT is wild type MB192. (b) Growth of MB192, Yp112-CMPT1, Yp112, and WT cells after $40 \mathrm{~h}$ growth in the presence of $60 \mathrm{mM} \mathrm{Pi}$. (c) The effect of varying the $\mathrm{pH}$ of the medium on the growth of MB192, Yp112-CMPT1, Yp112 and WT. (d) ${ }^{32} \mathrm{P}$ transport in Yp112-CmPT1 as a function of Pi concentration. A non-linear regression of Pi uptake by Yp112-CMPT1 at pH6 was used to estimate the $K_{m}$ of Pi uptake. OD(600): optical density measured at $600 \mathrm{~nm}$.

primary transformants was obtained by qRT-PCR, as described above. Two independent transgenic plants (CmPT1-Oe1 and CmPT1-Oe2), along with non-transformed 'Jinba' plants were exposed to either Pi-sufficient $(300 \mu \mathrm{M}$ $\mathrm{Pi}$; $+\mathrm{P})$ and $\mathrm{P}$-deficiency $(15 \mu \mathrm{M} \mathrm{Pi}$ - $\mathrm{P})$ conditions for 20 days. Each assay involved three replicates of a set of ten plants per genotype. Plant height, root volume, total dry biomass and the phosphate concentration in the whole plant were measured. The root volume was obtained by displacement [30]. Dry weight was obtained by weighing plants held at $80^{\circ} \mathrm{C}$ for three days. The whole plant phosphate concentration was estimated from a $\sim 0.1 \mathrm{~g}$ sample of dry matter, following [31].

(c)

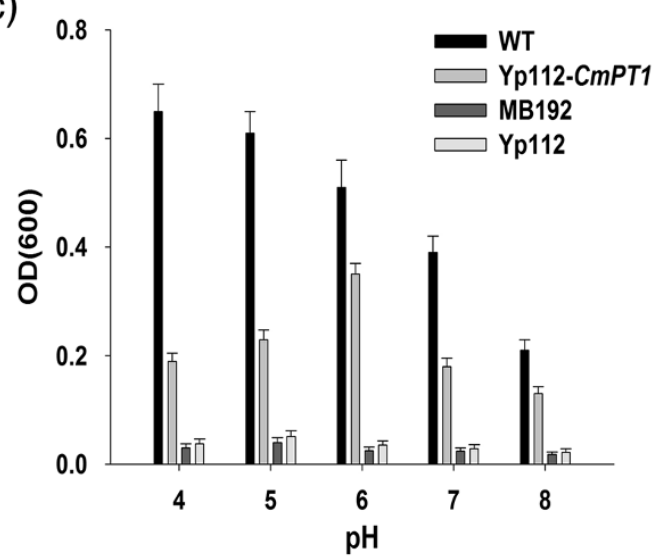

(d)

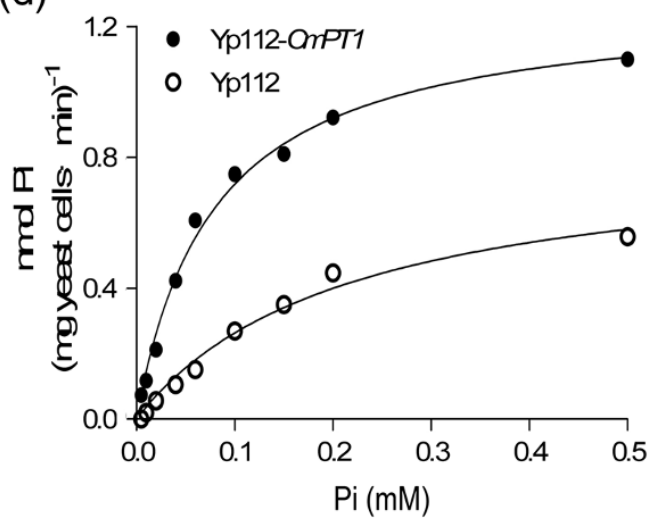

\section{Results}

\section{CmPT1 encodes a Pht1 Pi transporter}

The full-length CmPT1 cDNA was a 1,875 bp sequence, consisting of a 1,599 bp ORF, an 87 bp $5^{\prime}$-UTR and a 189 bp 3'-UTR (Figure 1). Its predicted translation product was a 532 residue polypeptide with a calculated molecular mass of $57.97 \mathrm{kDa}$ and a pI of 8.60. The protein contains 12 transmembrane domains with a large hydrophilic loop between TM6 and TM7 (Figure 1). The peptide sequence shares a high level of homology with known high affinity plant Pht1 proteins: $77.1 \%$ with AtPT1 (GenBank accession number AED94948), 78.5\% with LePT1 (AAB82146), 71.8\% with OsPT8 (Q8H6G8) and $78.9 \%$ with MtPT5 (ABM69111) (Figure 1). A phylogenetic analysis showed that the chrysanthemum sequence is closely related with other plant Pi transporters, and particularly with its tomato homolog LePT2 (AAB82147) (Figure 2). The Pht1 signature sequence GGDYPLSATIxSE [32] is present in CmPT1.

\section{Transcription of CmPT1}

CmPT1 transcription was strong in the root, weak in the stem, and below the level of detection in the leaf of plants exposed to either under both Pi-sufficient $(300 \mu \mathrm{M} \mathrm{Pi} ;+\mathrm{P})$ and Pi-deficient $(0 \mu \mathrm{M} \mathrm{Pi}$; $-\mathrm{P})$ conditions (Figure 3$)$. The relative transcript abundance in the root of $+\mathrm{P}$ and $-\mathrm{P}$ plants was, respectively, 35.5 and 100.0, and in the stem, respectively 2.1 and 3.1 . 

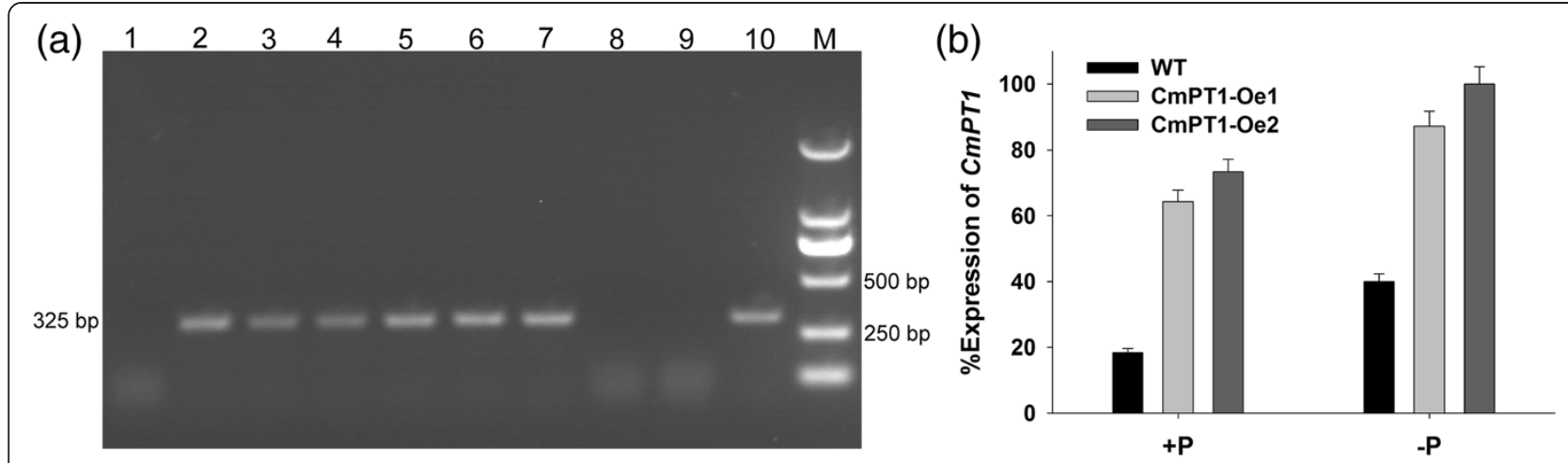

Figure $\mathbf{5}$ Validation of transgenic plants. (a) PCR analysis of genomic DNA extracted from kanamycin resistant regenerants. (b) Relative CmPT1 transcript abundance in wild type and transgenic plants.

\section{Complementation test in yeast}

Yeast strain MB192 cells heterologously expressing CmPT1 (Yp112-CmPT1) were able to survive at $0.02 \mathrm{mM}$ $\mathrm{Pi}$, and grew well at $0.06 \mathrm{mM} \mathrm{Pi}$; neither wild type (WT) cells, nor MB192 cells containing only Yp112 could do so.
At $0.1 \mathrm{mM} \mathrm{Pi}$, all cell types grew equally well (Figure 4a, b). Both WT and Yp112-CmPT1 cells grew faster at $\mathrm{pH} 4-6$ than at 7-8 (Figure 4c). The $\mathrm{pH}$ optimum for Yp112CmPT1 was around 6, whereas for the WT it was $4-5$. The results are consistent with the notion that $\mathrm{CmPT} 1$ operates

(a)

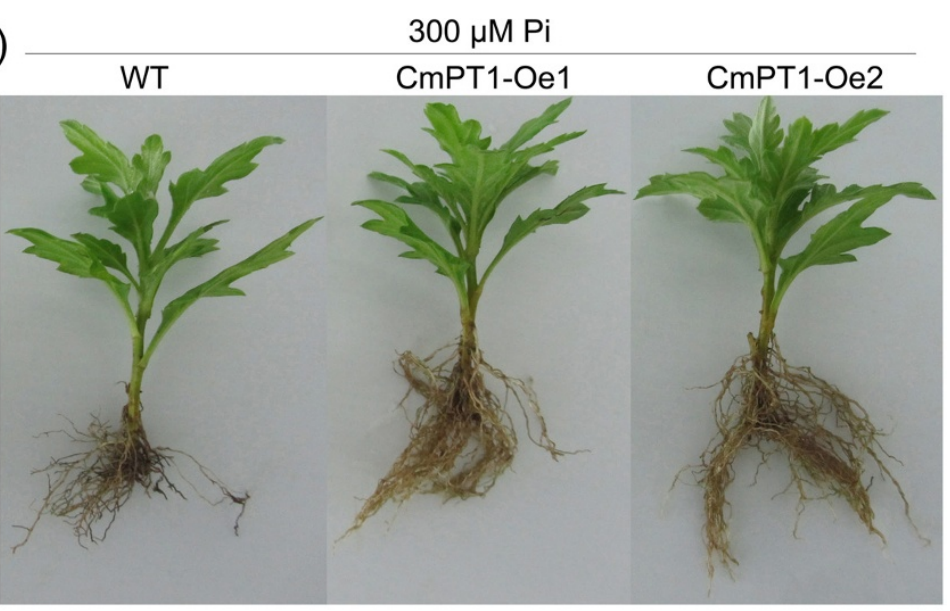

(b)
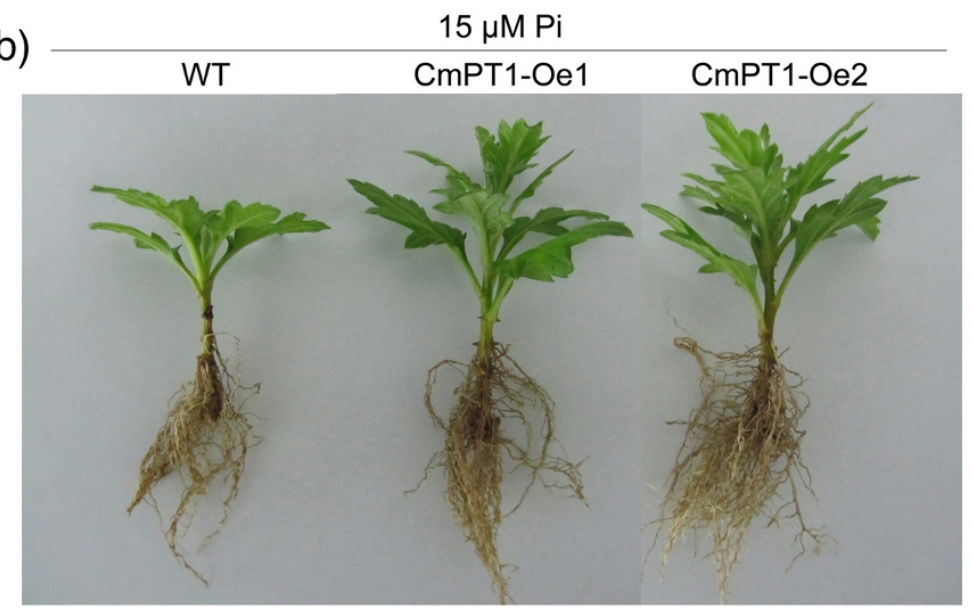

Figure 6 The effect on growth of constitutively expressing CmPT1. Wild type ('Jinba') and CmPT1 constitutive expression transgenics grown in the presence of (a) sufficient $\mathrm{Pi}\left("+\mathrm{P}^{\prime \prime}\right)$ and (b) deficient $\mathrm{Pi}($ ("-P") conditions. 
as an $\mathrm{H}^{+} / \mathrm{H}_{2} \mathrm{PO}_{4}$ symporter. To exclude interference from the activity of other PTs (particularly the low affinity ones), the dynamics of Pi transport of cells transformed with either p112A1NE-CmPT1 or the empty vector was monitored at $0.5 \mathrm{mM} \mathrm{Pi}$. The estimated mean $K_{m}$ of CmPT1 was $35.2 \mu \mathrm{M} \mathrm{Pi}$, as determined from three independent experiments (Figure 4d). Thus, CmPT1 is likely a high Pi affinity transporter, mediating Pi uptake in the micromolar range.

\section{CMPT1 constitutive expression in chrysanthemum} enhances tolerance to $\mathrm{Pi}$ deficiency

Six independent rooted presumptive CmPT1 transformants were selected by the genomic PCR assay (Figure 5a). When subjected to qRT-PCR, it was clear that the level of CmPT1 transcription in CmPT1-Oe1 and CmPT1-Oe2 was significantly higher than in the wild type plant both at Pi-sufficient $(300 \mu \mathrm{M} \mathrm{Pi}+\mathrm{P})$ and P-deficiency $(15 \mu \mathrm{M} \mathrm{Pi}$; -P) conditions (Figure 5b). The effect CmPT1 constitutive expression was to promote root growth and increase the accumulation of phosphate at $+\mathrm{P}$ (Figures 6 and 7), while at $-\mathrm{P}$, the plant height of the two transgenic plants was increased by respectively $38.7 \%$ and $35.5 \%$ over that of the wild type (Figure 7a); similarly, root volume in both transgenics was enhanced by, respectively, $53.3 \%$ and $66.7 \%$ (Figure $7 \mathrm{~b}$ ), dry weight by $37.1 \%$ and $48.6 \%$ and phosphate concentration by $54.4 \%$ and $68.7 \%$ (Figure $7 \mathrm{c}, \mathrm{d}$ ). Thus the constitutive expression of CmPT1 helped chrysanthemum plants to cope with phosphate deficiency.

\section{Discussion}

The uptake of $\mathrm{Pi}$ from a heterogeneous, constantly fluctuating environment is a major challenge faced by all plants. The process is largely governed by Pht1 transporters [2]. The predicted product of the chrysanthemum Pht1 homologue extracted from the cultivar 'Nannongyinshan' forms 12 transmembrane domains, separated into two groups of six by a charged hydrophilic loop (Figure 1). This structural arrangement is typical for Pht1 transporters [6]. The CmPT1 sequence shares a high level of sequence identity to other known high affinity $\mathrm{Pi}$ transporters and includes the signature sequence GGDYPLSATIxSE. A number of members of the Pht1 family are strongly expressed in the root, and are induced by phosphate starvation [10,13,14,16,33], just as was the case for CmPT1 (Figure 3a, b). Nevertheless its pattern of transcription is not completely the same as that of certain high affinity Pi transporters, in particular those of LePT1, which is transcribed in both the root and the leaf of tomato [16], and OsPT8 which is also transcribed in the rice grain [14]. CmPT1 transcript abundance was below the level of detection in the leaf, even in plants growing under phosphate deficient growing conditions, while its level in the stem was low but still discernible. The evidence as a whole strongly suggests that CmPT1 acts as a Pi transporter in chrysanthemum.

Several approaches have been used to investigate the Pi transport properties of the various PHT1 family members. In our study, CmPT1 was able to complement the
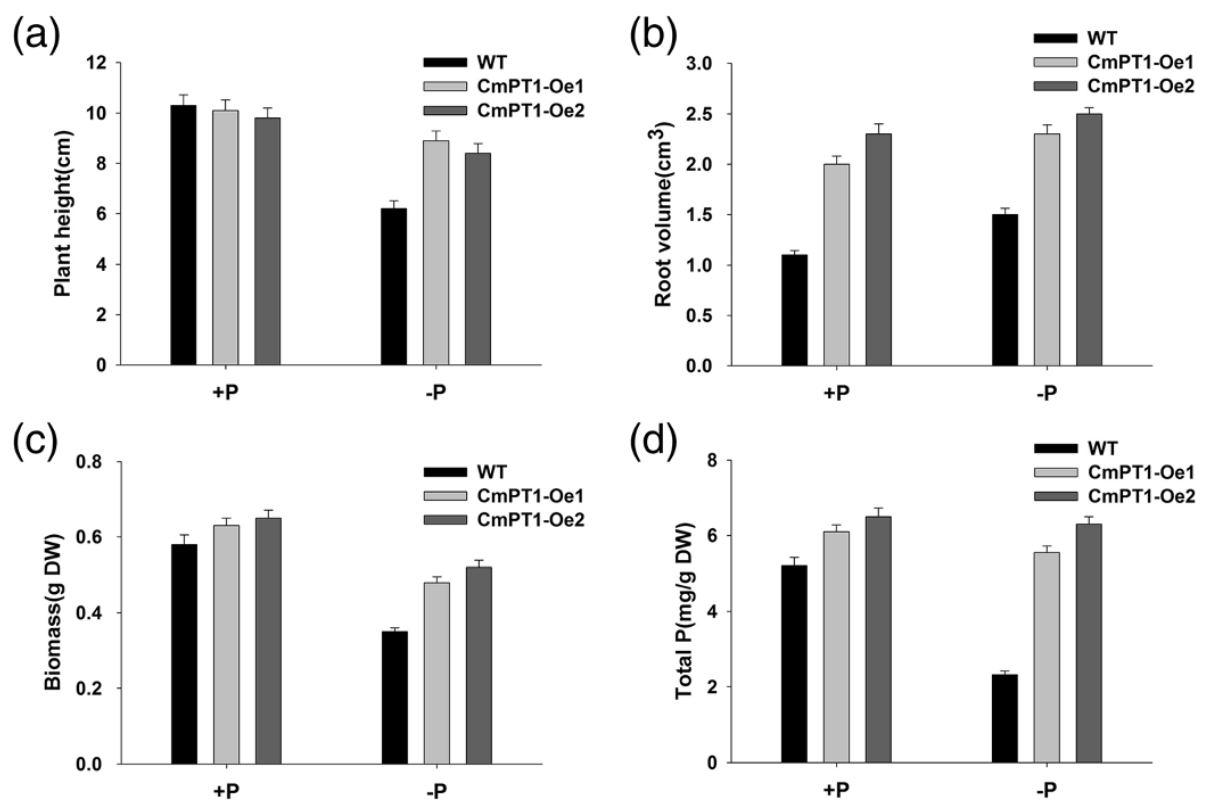

Figure 7 Phenotypic differences of the wild-type and CmPT1-overexpression lines. The effect of CmPT1 constitutive expression on (a) plant height, (b) root volume, (c) biomass accumulation and (d) phosphate accumulation. The data are expressed as means \pm standard error $(n=5)$. Columns bearing the same letter did not differ significantly from one another at $\mathrm{P}<0.01$. 
loss of high affinity Pi transporter activity of the yeast mutant MB192 in the high affinity concentration range (Figure 4). Its estimated $\mathrm{Km}$ of $35.2 \mu \mathrm{M}$ (Figure $4 \mathrm{~d}$ ) lies within the $K_{m}$ range of other plant high affinity phosphate transporters, measured by a similar method - the range being from $13 \mu \mathrm{M}$ to $97 \mu \mathrm{M}[13,14,21]$. Heterologous expression in yeast provides an informative means of characterizing plant proteins, but the system does have some limitations with respect to assessing the kinetics of plant phosphate transporters, the yeast complementation only partially reflects the behavior of PHT1 proteins in plants $[2,20]$. Estimates of $K_{m}$ obtained by transcription in suspension cultures are substantially lower: $3.1 \mu \mathrm{M}$ for $A$. thaliana Pht1 when transcribed in tobacco cells [34]; $9.1 \mu \mathrm{M}$ for the barley protein HORvu; Pht1;1 when expressed in rice cells [20]. It may be possible to obtain a more accurate estimate of $\mathrm{Km}$ to CmPT1 by using a homologous system in the future.

Transcription of the gene encoding the primary transporter is often considered to be the control point in pathways of nutrient uptake [35]. Many efforts have been made to research the function of the $P T$ genes and the possibility of using the $P T$ genes to improve the adaptability to Pi-deficient stress of crops. Overexpression of an Arabidopsis thaliana high-affinity phosphate transporter gene in tobacco cultured cells enhances cell growth under phosphate-limited conditions [34]. Suspension cells of transgenic rice that over-expressed a high-affinity phosphate transporter $H v P T 1$ were able to take up phosphate at a much higher rate than control cells [20], but over-expression of the same gene in transgenic barley plants does not enhance phosphate uptake rates at the whole plant level [36]. Park transferred a high-affinity phosphate transporter gene from tobacco (NtPT1) to rice enhanced phosphate uptake and accumulation under low-P condition [37]. Over-expressing low affinity phosphate transporter genes OsPT1 and OsPT2 in rice cannot bring significant differences to the transgenic plants under Pi-deficient supply $[15,38]$. Here, a Pht1 transporter gene which cloned from a low P tolerant cultivar of cut chrysanthemum 'Nannongyinshan' transferred to 'Jinba', a low $\mathrm{P}$ sensitive cultivar, the growth of transgenic plants was significantly improved over that of the non-transformed ones under Pi deficient conditions (Figures 6 and 7). Considering the two cultivars sharing the same sequence of CmPT1 and the transgenic and non-transgenic plants having same genetic background, physiological and biochemical changes are direct results of the overexpression of CmPT1 gene. Two possibilities can be envisaged to explain mechanisms of these changes. More copies of the transporter protein are inserted into the plasma membrane in the overexpression transgenic lines. Evidence for the transcriptional control of PT activity has been shown by parallel increases in PT transcript and protein levels detected by high-affinity PT antibody in tomato [39]. The CmPT1 gene may participate in the Pi-starvation signal transduction pathways, Overexpression of the CmPT1 gene directly or indirectly caused Pi-dependent root architecture alteration with enhanced root elongation and proliferated lateral root growth (Figures 6 and $7 \mathrm{~b}$ ), these responses enable the optimization of soil exploration and of the absorptive surface area of the root [40].

\section{Conclusion}

In conclusion, CmPT1 encodes a typical, root-expressed, high affinity phosphate transporter in chrysanthemum. Taking the flexible expression pattern into account, CmPT1 might function in a wide range of Pi environments and play a significant role in phosphate acquisition under natural conditions.

\section{Competing interests}

The authors declare that they have no competing interests.

\section{Authors' contributions}

$\mathrm{PL}$ and $\mathrm{AS}$ performed the experiments; PL wrote the manuscript; SC, SZ, ZG and JJ edited the manuscript; WF, SC, YL and JJ revised the manuscript; JJ and FC designed the experiments. All authors read and approved the final manuscript.

\section{Acknowledgements}

We thank Professor Guohua Xu (College of Resources and Environmental Sciences, Nanjing Agricultural University) for offer the yeast strains. The study was supported by the National Natural Science Foundation of China (31272202), the Program for New Century Excellent Talents in University of the Chinese Ministry of Education (NCET-10-0492, NCET-12-0890), the Program for Sci-Tech Support Research, Jiangsu, China, (BE2012350), the Fundamental Research Funds for the Central Universities (KYZ201147) from Nanjing Agricultural University and Youth Science and Technology Innovation Fund from Nanjing Agricultural University( KJ2011009).

Received: 20 September 2013 Accepted: 6 January 2014

Published: 10 January 2014

\section{References}

1. Schachtman DP, Reid RJ, Ayling S: Phosphorus uptake by plants: from soil to cell. Plant Physiol 1998, 116:447-453.

2. Nussaume L, Kanno S, Javot H, Marin E, Nakanishi TM, Thibaud MC: Frontiers: Phosphate import in plants: focus on the PHT1 transporters. Front Plant Traffic Transp 2011, 2:1-8.

3. Shin $H$, Shin HS, Dewbre GR, Harrison MJ: Phosphate transport in Arabidopsis: Pht1;1and Pht1;4 play a major role in phosphate acquisition from both low- and high-phosphate environments. Plant J 2004, 39:629-642.

4. Holford I: Soil phosphorus: its measurement, and its uptake by plants. Aust J Soil Res 1997, 35:227-240.

5. Oelkers E, Valsami-Jones E: Phosphate mineral reactivity and global sustainability. Elem 2008, 4:83-88.

6. Raghothama K: Phosphate acquisition. Annu Rev Plant Biol Plant Mol Biol 1999, 50:665-693.

7. Bieleski R: Phosphate pools, phosphate transport, and phosphate availability. Ann Rev Plant Physiol 1973, 24:225-252.

8. Raghothama K: Phosphate transport and signaling. Curr Opin Plant Biol 2000, 3:182-187.

9. Furihata T, Suzuki M, Sakurai H: Kinetic characterization of two phosphate uptake systems with different affinities in suspension-cultured Catharanthusroseus protoplasts. Plant Cell Physiol 1992, 33:1151-1157. 
10. Muchhal US, Pardo JM, Raghothama KG: Phosphate transporters from the higher plant Arabidopsis thaliana. Proc Natl Acad Sci USA 1996 93:10519-10523.

11. Misson J, Thibaud MC, Bechtold N, Raghothama K, Nussaume L: Transcriptional regulation and functional properties of Arabidopsis Pht1; 4 , a high affinity transporter contributing greatly to phosphate uptake in phosphate deprived plants. Plant Mol Biol 2004, 55:727-741.

12. Catarecha P, Segura MD, Franco-Zorrilla JM, García-Ponce B, Lanza M, Solano R, Paz-Ares J, Leyva A: A mutant of the Arabidopsis phosphate transporter PHT1; 1 displays enhanced arsenic accumulation. Plant Cell Online 2007, 19:1123-1133.

13. Ai P, Sun S, Zhao J, Fan X, Xin W, Guo Q, Yu L, Shen Q, Wu P, Miller A: Two rice phosphate transporters, OsPht $1 ; 2$ and OsPht $1 ; 6$, have different functions and kinetic properties in uptake and translocation. Plant 2009, 57:798-809.

14. Jia H, Ren H, Gu M, Zhao J, Sun S, Zhang X, Chen J, Wu P, Xu G: The phosphate transporter gene OsPht 1; 8 is involved in phosphate homeostasis in rice. Plant Physiol 2011, 156:1164-1175.

15. Sun S, Gu M, Cao Y, Huang X, Zhang X, Ai P, Zhao J, Fan X, Xu G: A constitutive expressed phosphate transporter, OsPht1; 1 , modulates phosphate uptake and translocation in phosphate-replete rice. Plant Physiol 2012, 159:1571-1581.

16. Liu C, Muchhal US, Uthappa M, Kononowicz AK, Raghothama KG: Tomato phosphate transporter genes are differentially regulated in plant tissues by phosphorus. Plant Physiol 1998, 116:91-99.

17. Baek S, Chung I, Yun S: Molecular cloning and characterization of a tobacco leaf cDNA encoding a phosphate transporter. Mol Cells 2001, 11:1-6.

18. Kai M, Takazumi K, Adachi H, Wasaki J, Shinano T, Osaki M: Cloning and characterization of four phosphate transporter cDNAs in tobacco. Plant Sci 2002, 163:837-846.

19. Nagy R, Vasconcelos M, Zhao S, McElver J, Bruce W, Amrhein N, Raghothama K, Bucher M: Differential regulation of five Pht1 phosphate transporters from maize (Zea mays L.). Plant Biol 2006, 8:186-197.

20. Rae AL, Cybinski DH, Jarmey JM, Smith FW: Characterization of two phosphate transporters from barley; evidence for diverse function and kinetic properties among members of the Pht1 family. Plant Mol Biol 2003, 53:27-36.

21. Liu J, Versaw WK, Pumplin N, Gomez SK, Blaylock LA, Harrison MJ: Closely related members of the Medicago truncatula PHT1 phosphate transporter gene family encode phosphate transporters with distinct biochemical activities. J Biol Chem 2008, 283:24673-24681.

22. Loth-Pereda V, Orsini E, Courty PE, Lota F, Kohler A, Diss L, Blaudez D, Chalot M, Nehls U, Bucher M: Structure and expression profile of the phosphate Pht1 transporter gene family in mycorrhizal Populus trichocarpa. Plant Physiol 2011, 156:2141-2154.

23. Fan C, Wang $X$, Hu R, Wang Y, Xiao C, Jiang Y, Zhang X, Zheng C, Fu Y: The pattern of Phosphate transporter 1 genes evolutionary divergence in Glycine max L. BMC Plant Biol 2013, 13(1):48.

24. Liu P, Chen SM, Fang WM, Jiang JF, Guan ZY, Chen FD: Preliminary evaluation on tolerance to phosphorous deficiency of 32 cultivars of cut chrysanthemum. Acta Ecol Sin 2013, 33(21):6863-6868.

25. Jones JB Jr: Hydroponics: its history and use in plant nutrition studies. J Plant Nutr 1982, 5:1003-1030.

26. Song A, Lu J, Jiang J, Chen S, Guan Z, Fang W, Chen F: Isolation and characterisation of Chrysanthemum crassum SOS1, encoding a putative plasma membrane $\mathrm{Na}^{+} / \mathrm{H}^{+}$antiporter. Plant Biol 2012, 14:706-713.

27. Livak KJ, Schmittgen TD: Analysis of relative gene expression data using real-time quantitative PCR and the $2^{-\Delta \Delta C}$. Method 2001, 25:402-408.

28. Hofgen R, Willmitzer L: Storage of competent cells for Agrobacterium transformation. Nucleic Acids Res 1988, 16:9877-9877

29. Shinoyama H, Komano M, Nomura Y, Nagai T: Introduction of Delta-endotoxin Gene of Bacillus thuringiensis to Chrysanthemum [Dendranthema $x$ grandiflorum (Ramat.) Kitamura] for Insect Resistance. Breed Sci 2002, 52:43-50.

30. Burdett A: A nondestructive method for measuring the volume of intact plant parts. Can J Forest Res 1979, 9:120-122.

31. Chen A, Hu J, Sun S, Xu G: Conservation and divergence of both phosphate-and mycorrhiza-regulated physiological responses and expression patterns of phosphate transporters in solanaceous species. New Phytol 2007, 173:817-831.
32. Karandashov $V$, Bucher M: Symbiotic phosphate transport in arbuscular mycorrhizas. Trends Plant Sci 2005, 10:22-29.

33. Wu Z, Zhao J, Gao R, Hu G, Gai J, Xu G, Xing H: Molecular Cloning, Characterization and Expression Analysis of Two Members of the Pht1 Family of Phosphate Transporters in Glycine max. PLoS One 2011, 6:e19752.

34. Mitsukawa N, Okumura S, Shirano Y, Sato S, Kato T, Harashima S, Shibata D: Overexpression of an Arabidopsis thaliana high-affinity phosphate transporter gene in tobacco cultured cells enhances cell growth under phosphate-limited conditions. Proc Natl Acad Sci USA 1997, 94:7098-7102.

35. Kunze R, Frommer WB, Flügge Ul: Metabolic engineering of plants: the role of membrane transport. Metab Eng 2002, 4:57-66.

36. Rae A, Jarmey J, Mudge S, Smith F: Over-expression of a high-affinity phosphate transporter in transgenic barley plants does not enhance phosphate uptake rates. Funct Plant Biol 2004, 31:141-148.

37. Park M, Baek S, de los Reyes B, Yun S: Overexpression of a high-affinity phosphate transporter gene from tobacco (NtPT1) enhances phosphate uptake and accumulation in transgenic rice plants. Plant Soil 2007 292:259-269.

38. Liu F, Wang Z, Re H, Shen C, Li Y, Ling HQ, Wu C, Lian X, Wu P: OsSPX1 suppresses the function of OsPHR2 in the regulation of expression of OsPT2 and phosphate homeostasis in shoots of rice. Plant J 2010, 62:508-517.

39. Muchhal US, Raghothama KG: Transcriptional regulation of plant phosphate transporters. Proc Natl Acad Sci USA 1999, 96:5868-5872.

40. López-Bucio J, Cruz-Ramirez A, Herrera-Estrella L: The role of nutrient availability in regulating root architecture. Curr Opin Plant Biol 2003, 6:280-287.

doi:10.1186/1471-2229-14-18

Cite this article as: Liu et al: A putative high affinity phosphate

transporter, CmPT1, enhances tolerance to Pi deficiency

of chrysanthemum. BMC Plant Biology 2014 14:18.

\section{Submit your next manuscript to BioMed Central and take full advantage of:}

- Convenient online submission

- Thorough peer review

- No space constraints or color figure charges

- Immediate publication on acceptance

- Inclusion in PubMed, CAS, Scopus and Google Scholar

- Research which is freely available for redistribution 